\title{
A nonparametric unit root test under nonstationary volatility
}

\author{
Burak Alparslan Eroğlu, Taner Yiğit* \\ Department of Economics, Bilkent University, Ankara, 06800, Turkey
}

\section{H I G H L I G H T S}

- A nonparametric unit root test robust to nonstationary volatility is proposed.

- The proposed test statistic does not require a correction of serial correlation.

- The proposed test is correctly sized and has desirable power.

- In finite sample properties, our test outperforms other tests in the literature.

\section{A R T I C L E I N F O}

\section{Article history:}

Received 18 September 2015

Received in revised form

25 December 2015

Accepted 10 January 2016

Available online 15 January 2016

\section{JEL classification:}

C2

C12

C14

\section{Keywords:}

Nonstationary volatility

Fractionally integrated time series

Variance ratio statistic

Unit root testing

\begin{abstract}
A B S T R A C T
We develop a new nonparametric unit root testing method that is robust to permanent shifts in innovation variance. Unlike other methods in the literature, our test does not require a parametric specification or lag/bandwidth selection to adjust for serial correlation.
\end{abstract}

(c) 2016 Elsevier B.V. All rights reserved.

\section{Introduction}

Recent body of empirical evidence indicates that variance shifts (nonstationary volatility) is a common occurrence in macroeconomic and financial data; see Busetti and Taylor (2003), McConnell and Perez-Quiros (1998) and Sensier and Van Dijk (2004). This finding coupled with nonstationarity in the levels of these types of data led the researchers to investigate the impact of variance shifts on unit root tests. In one of these studies, Cavaliere and Taylor (2007), henceforth CT, document that under nonstationary volatility, the asymptotic distributions of standard unit root tests are altered by the inclusion of a new nuisance parameter called the "variance profile", leading to size distortions in these tests. In order to achieve correct inference, CT suggest first consistently estimating this nuisance parameter and then updating the asymptotic

\footnotetext{
* Corresponding author. Tel.: +90 3122901898.

E-mail address: tyigit@bilkent.edu.tr (T. Yiğit).
}

distribution of Phillips and Perron's (1988) tests with this estimate. While their inclusion of the new nuisance parameter generates significant gains in size over classical unit root tests, they still rely on the methodologies used in earlier studies to correct for other nuisance parameters such as serial correlation in errors. CT adjust their test statistic via the estimation of the long run variance, obtained by a semi-parametric kernel or a parametric ADF based regression estimation. The success of these methods highly depends on lag length, bandwidth and Kernel selection in terms of finite sample properties. In this paper, we propose a nonparametric unit root test that is robust to nonstationary volatility problem yet does not require a long run variance estimation.

We derive our test statistic by modifying Nielsen's (2009) nonparametric variance ratio statistic with the nonparametric variance profile estimator of CT. Computation of the proposed test statistic involves a fractional transformation of observed series, but it does not require any parametric regression or the choice of any tuning parameters like lag length and bandwidth. Therefore, we not only modify Nielsen's test to be robust against nonstationary volatility, but also improve on the finite sample properties of CT 
statistic for all considered types of serial correlation. Derivation of the limiting distribution of fractionally integrated processes with nonstationary volatility and the proofs are placed in Appendix. ${ }^{1}$

\section{Model and variance ratio test}

\subsection{Model}

Let $\left\{x_{t}\right\}_{t=0}^{T}$ be generated by:

$x_{t}=y_{t}+\theta^{\prime} \delta_{t}$

$y_{t}=\rho y_{t-1}+u_{t}$

$u_{t}=C(L) \varepsilon_{t}$

$\varepsilon_{t}=\sigma_{t} e_{t}$

where $e_{t} \sim$ i.i.d. $(0,1)$ and $\theta^{\prime} \delta_{t}$ is the deterministic term and $C(L)$ is the lag polynomial. From CT, we have following assumptions:

Assumption. $A$ A. 1 The lag polynomial $C(L) \neq 0$ for all $|L| \leq 1$, and $\sum_{j=0}^{\infty} j\left|c_{j}\right|<\infty$. $\mathbb{E}\left|e_{t}\right|^{r}<K<\infty$ for some $r \geq 4$.

A.2 $\rho$ satisfies $|\rho| \leq 1$.

$\mathcal{A} .3 \sigma_{t}$ satisfies $\sigma_{\lfloor T s\rfloor}:=\omega(s)$ for all $s \in[0,1]$, where $\omega(.) \in \mathscr{D}$ is non-stochastic and strictly positive. For $t<0, \sigma_{t}$ is uniformly bounded, that is there exists a $\sigma^{*}$ such that $\sigma_{t} \leq \sigma^{*}<\infty$.

The assumptions $\mathcal{A} .1$ and $\mathcal{A} .2$ are very standard in unit root testing literature. CT characterize the dynamics of innovation variance in $\mathcal{A}$.3, which should be bounded and display a countable number of jumps.

A fundamental object that is defined in CT is given below:

$\eta(s):=\left(\int_{0}^{1} \omega(r)^{2} d r\right)^{-1}\left(\int_{0}^{s} \omega(r)^{2} d r\right)$.

This object is referred to as the variance profile of the process. Further, CT show that $\int_{0}^{1} \omega(r)^{2} d r=\bar{\omega}^{2}$ is the limit of $T^{-1} \sum_{t=1}^{T} \sigma_{t}^{2}$.

\subsection{Variance Ratio test under nonstationary volatility}

So as to modify the Variance Ratio test (Nielsen, 2009) statistic we first need the fractional partial sum operator for some $d>0$ :

$$
\begin{aligned}
\tilde{x}_{t} & :=\Delta_{+}^{-d} x_{t}=(1-L)_{+}^{-d} x_{t}=\sum_{k=0}^{t-1} \frac{\Gamma(k+d)}{\Gamma(d) \Gamma(k+1)} x_{t-k} \\
& =\sum_{k=0}^{t-k} \pi_{k}(d) x_{t-k}
\end{aligned}
$$

where $\Gamma$ (.) is gamma function. Under the assumptions $\mathcal{A}$, following lemmas hold:

Lemma 1. Assume that $\left\{u_{t}\right\}_{t=0}^{T}$ is generated by (3)-(4) and $\rho=$ $1-c / T$ with $c \geq 0$.

i. $y_{T}(t)=T^{-1 / 2} \sum_{k=1}^{\lfloor T t\rfloor} e^{-c(\lfloor T t\rfloor-k)} u_{k} \stackrel{w}{\longrightarrow} \bar{\omega} C(1) J_{\omega}^{c}(t)$, where $J_{\omega}^{c}(t)=\int_{0}^{t} \exp (-c(t-s)) d B_{\omega}(s)$ and $B_{\omega}(s)=\bar{\omega}^{-1} \int_{0}^{s} \omega(r) d B(r)$.

ii. $B_{\omega}(s)=B_{\eta}(s):=B(\eta(s))$ where $B_{\eta}(s)$ variance transformed Brownian motion, $\eta(s)$ is defined in (5). Thus, $J_{\eta}^{c}(t):=J_{\omega}^{c}(t)=$ $\int_{0}^{t} \exp (-c(t-s)) d B_{\eta}(s)$.

iii. For all $d>0, \tilde{y}_{T}(t)=T^{-d} \Delta_{+}^{-d} y_{T}(t) \stackrel{w}{\longrightarrow} \bar{\omega} C(1) J_{\omega, d}^{c}(t)$, where $J_{\omega, d}^{c}(t)=\Gamma(d+1)^{-1} \int_{0}^{t}(t-s)^{d} d J_{\omega}^{c}(s)$. Further, we have $J_{\omega, d}^{c}(t)=J_{\eta, d}^{c}(t)$.

\footnotetext{
1 The notation in the paper follows Cavaliere and Taylor (2007).
}

Remark 1. Lemma 1(i) and (ii) are from Cavaliere (2005) and CT. Lemma 1(iii) is new and establishes weak convergence for fractionally integrated processes with non-stationary volatility. Although Demetrescu and Sibbertsen (2014) model the fractional integrated process with non-stationary volatility, they do not establish weak convergence of this object.

Remark 2. Note that under the null hypothesis of $\rho=1$ or $c=$ 0 the above variance transformed Uhlenbeck-Ornstein process becomes a variance transformed Brownian motion. For instance, under the null the partial sum process $\tilde{y}_{T}(t)$ will converge to $\bar{\omega} C(1) \int_{0}^{t}(t-s)^{d} d B_{\eta}(s)$ where we can define $B_{\eta, d}(t):=\int_{0}^{t}(t-$ $s)^{d} d B_{\eta}(s)$. This limiting distribution resembles the type II fractional Brownian motions defined by Marinucci and Robinson (2000), since $B_{\eta, d}(t)$ does not contain any pre-historic influence (see also Wang et al., 2002).

Like Nielsen (2009), we apply OLS detrending to the observed series $x_{t}$ to clean out the deterministic terms. Let $\hat{x}_{t}$ be the OLS detrended residuals and defining $\tilde{\hat{x}}_{t}=\Delta_{+}^{-d} \hat{x}_{t}$, our test statistic is then given by:

$\tau_{\eta}(d)=T^{2 d} \frac{\sum_{t=1}^{T} \hat{x}_{t}^{2}}{\sum_{t=1}^{T} \tilde{\hat{x}}_{t}^{2}}$.

Theorem 1. Assume that the time series $\left\{x_{t}\right\}$ is generated by Eqs. (1)(4) and $\rho=1-c / T$ for $c \geq 0$. Let $j=0$ when $\delta_{t}=0, j=1$ when $\delta_{t}=1$ and when $\delta_{t}=[1, t]^{\prime}$ for $d>0$

$$
\begin{aligned}
\text { i. } & \hat{x}_{T}(t) \stackrel{w}{\longrightarrow} J_{\eta, j}^{c}(t) \text { where } J_{\eta, j}^{c}(t)=J_{\eta}^{c}(t)-\left(\int_{0}^{1} J_{\eta}^{c}(s) D_{j}(s)^{\prime} d s\right) \\
& \left(\int_{0}^{1} D_{j}(s) D_{j}(s)^{\prime} d s\right)^{-1} D_{j}(t) \text { for } j=1,2 \text {, and } D_{1}(s)=1, D_{2}(s)= \\
& {[1, s]^{\prime} \text { and } J_{\eta, 0}^{c}(t)=J_{\eta}^{c}(t) \text {. } }
\end{aligned}
$$

ii. $\tilde{\hat{x}}_{T}(t) \stackrel{w}{\longrightarrow} J_{\eta, d, j}^{c}(t)$ where $J_{\eta, d, j}^{c}(t)=J_{\eta, d}^{c}(t)-\left(\int_{0}^{1} J_{\eta, d}^{c}(s) D_{j}\right.$ $\left.(s)^{\prime} d s\right)\left(\int_{0}^{1} D_{j}(s) D_{j}(s)^{\prime} d s\right)^{-1} \int_{0}^{t} \frac{(t-r)^{d-1}}{\Gamma(d)} D_{j}(r) d r$ for $j=1,2$. Further $J_{\eta, d, 0}^{c}(t)=J_{\eta, d}^{c}(t)$.

iii. $\tau_{\eta}(d)=T^{2 d} \frac{\sum_{t=1}^{T} \hat{x}_{t}^{2}}{\sum_{t=1}^{T} \tilde{\hat{x}}_{t}^{2}} \stackrel{w}{\longrightarrow} U_{j, \eta}(d)=\frac{(\bar{\omega} C(1))^{2} \int_{0}^{1} J_{\eta, j}^{c}(s)^{2} d s}{(\bar{\omega} C(1))^{2} \int_{0}^{1} J_{\eta, d, j}^{c}(s)^{2} d s}=$ $\frac{\int_{0}^{1} J_{\eta, j}^{c}(s)^{2} d s}{\int_{0}^{1} J_{\eta, d, j}^{c}(s)^{2} d s}$.

Remark 3. Note that short run dynamics cancel out in asymptotic distribution since the numerator and the denominator share the same long run variance component in part (iii).

\subsection{Simulated asymptotic distribution}

The test statistic obtained in Theorem 1 involves $\eta(s)$ as nuisance parameter which can be consistently estimated by modifying the nonparametric estimator in $\mathrm{CT}$ :

$\hat{\eta}(s):=\frac{\sum_{t=1}^{\lfloor s\rfloor}\left(\Delta \hat{x}_{t}\right)^{2}+(T s-\lfloor T s\rfloor)\left(\Delta \hat{x}_{\lfloor T s\rfloor+1}\right)^{2}}{\sum_{t=1}^{T}\left(\Delta \hat{x}_{t}\right)^{2}}$.

Theorem 2. Under the conditions of Theorem 1

i. (CT show) $B_{\hat{\eta}, T}(s):=T^{-1 / 2} \sum_{t=1}^{\lfloor(\hat{\eta}\lfloor T s\rfloor / T) T\rfloor} e_{t} \stackrel{w}{\longrightarrow} B_{\eta}(s)$.

ii. $B_{\hat{\eta}, d, T}(s):=T^{-d} \Delta_{+}^{-d} B_{\hat{\eta}, T}(s) \stackrel{\sum_{w}^{t=1}}{\longrightarrow} B_{\eta, d}(s)$. 
Table 1

Empirical size and power with no serial correlation.

\begin{tabular}{|c|c|c|c|c|c|c|c|}
\hline & & \multicolumn{3}{|l|}{$\tau_{\eta}(d)$} & \multicolumn{3}{|l|}{$M Z_{t}^{S}$} \\
\hline$C V$ & $T=100$ & 0.052 & 0.251 & 0.598 & 0.034 & 0.171 & 0.527 \\
\hline \multirow[t]{2}{*}{$S B V$} & $T=100$ & 0.052 & 0.284 & 0.656 & 0.031 & 0.311 & 0.738 \\
\hline & $T=500$ & 0.050 & 0.993 & 1.000 & 0.046 & 1.000 & 1.000 \\
\hline$T V$ & $T=100$ & 0.054 & 0.257 & 0.588 & 0.029 & 0.261 & 0.654 \\
\hline \multirow[t]{2}{*}{ EISV } & $T=100$ & 0.061 & 0.280 & 0.633 & 0.034 & 0.300 & 0.687 \\
\hline & $T=500$ & 0.053 & 0.949 & 1.000 & 0.044 & 0.986 & 1.000 \\
\hline
\end{tabular}

Table 2

Empirical size and power with $\mathrm{AR}(1)$ innovations.

\begin{tabular}{|c|c|c|c|c|c|c|c|}
\hline & & \multicolumn{3}{|l|}{$\underline{\tau_{\eta}(d)}$} & \multicolumn{3}{|l|}{$\underline{M Z_{t}^{S}}$} \\
\hline$C V$ & $T=100$ & 0.016 & 0.237 & 0.507 & 0.035 & 0.140 & 0.290 \\
\hline \multirow[t]{2}{*}{$S B V$} & $T=100$ & 0.018 & 0.260 & 0.559 & 0.038 & 0.224 & 0.408 \\
\hline & $T=500$ & 0.043 & 0.982 & 1.000 & 0.041 & 0.976 & 0.995 \\
\hline$T V$ & $T=100$ & 0.016 & 0.235 & 0.510 & 0.036 & 0.186 & 0.321 \\
\hline \multirow[t]{2}{*}{ EISV } & $T=100$ & 0.017 & 0.175 & 0.419 & 0.014 & 0.079 & 0.182 \\
\hline & $T=500$ & 0.045 & 0.977 & 1.000 & 0.043 & 0.934 & 0.987 \\
\hline
\end{tabular}

Table 3

Empirical size and power with ARMA(2,2)innovations.

\begin{tabular}{|c|c|c|c|c|c|c|c|}
\hline & & \multicolumn{3}{|l|}{$\underline{\tau_{\eta}(d)}$} & \multicolumn{3}{|l|}{$M Z_{t}^{S}$} \\
\hline & & $\rho=1$ & $\rho=0.93$ & $\rho=0.86$ & $\rho=1$ & $\rho=0.93$ & $\rho=0.86$ \\
\hline$C V$ & $T=100$ & 0.047 & 0.240 & 0.591 & 0.020 & 0.071 & 0.218 \\
\hline \multirow[t]{2}{*}{ SBV } & $T=100$ & 0.049 & 0.286 & 0.656 & 0.011 & 0.215 & 0.490 \\
\hline & $T=500$ & 0.048 & 0.987 & 1.000 & 0.044 & 0.966 & 0.995 \\
\hline$T V$ & $T=100$ & 0.047 & 0.269 & 0.607 & 0.014 & 0.144 & 0.332 \\
\hline EISV & $T=500$ & 0.049 & 0.884 & 0.996 & 0.046 & 0.654 & 0.868 \\
\hline
\end{tabular}

After obtaining the consistent estimate for $\eta(s)$, we can simulate the asymptotic distribution and the critical value for the test statistic. First, we choose a step level $N$. For $s=j / N$ for $j=$ $1,2, \ldots, N$, then we compute $\hat{\eta}(\lfloor T s\rfloor / T)$ using (8). By drawing $e_{t}$ from $N(0,1)$, we obtain $B_{\hat{\eta}, T}(s)$. Then applying fractional integration operator $\Delta_{+}^{-d}$ to this object and multiplying it by $T^{-d}$, we get $B_{\hat{\eta}, d, T}(s)$. Applying appropriate demeaning and detrending procedure to this object we obtain the asymptotic distribution for the test statistic. This asymptotic distribution is then used to generate the critical values for the test. The proposed test rejects the null hypothesis for large values the test statistic, that is, we reject if $\tau_{\eta}(d)$ is greater than $(1-\alpha)$ quantile of $U_{\eta, j}$.

\section{Monte Carlo experiments}

In the Monte Carlo simulations, data is generated according to Eqs. (1)-(4) with $T=\{100,500\}$. We consider following specifications for error term variance:

i. Constant volatility (CV): $\omega(s)=1$ for $s \in[0,1]$.

ii. Single break in volatility (SBV): $\omega(s)=1+2 * 1(s>0.2 * T)$ for $s \in[0,1]$.

iii. Trending volatility (TV): $\omega(s)=1+2 * s$ for $s \in[0,1]$.

iv. Exponential integrated Stochastic volatility (EISV): $\omega(s)=$ $\exp (4 B(s))$ for $s \in[0,1]$ where $B(s)$ is standard Brownian process.

The innovations $e_{t}$ are drawn from $N(0,1)$. All simulations are conducted $M C=10000$ times. We fix the step size $N$ to $T$ in simulating the variance shifted Brownian motions. We consider four scenarios for serial correlation in innovations. First one does not contain any serial correlation. In second, $u_{t}$ follows a simple AR(1) model: $u_{t}=0.5 u_{t-1}+\varepsilon_{t}$, third is an ARMA(2,2) process: $u_{t}=0.1 u_{t-1}+0.07 u_{t-2}-0.4 \varepsilon_{t-1}+0.2 \varepsilon_{t-2}+\varepsilon_{t}$. Last one follows a MA(2) process; $u_{t}=-0.2 \varepsilon_{t-1}+0.15 \varepsilon_{t-2}$. We fix $\rho=$ $\{1,0.93,0.86\} . \rho=0$ indicates size and other values are for power evaluation. We also provide simulation for Cavaliere and Taylor (2007) $M Z_{t}^{S}$ test.

Remark 4. The simulation scenario (iv) is not covered by the assumption $\mathcal{A} .3$, as nonstationary volatility is stochastic. However, the simulations show that in this case our procedure is working well (see Tables 1-4).

\section{Conclusion}

Simulation evidence suggests the proposed nonparametric unit root test has desirable size and power properties in all scenarios considered. Our test almost dominates CT's test in terms of size. Furthermore, finite sample power results of our test are better than CT's tests except for the case of no serial correlation.

\footnotetext{
2 The confidence level is 0.05 and all data is demeaned. $d$ is fixed to 0.1 as recommended in Nielsen (2009). For formula and asymptotic distribution of $M Z^{s}$ test see CT. In fact, CT propose 3 different test statistic, but we only give the results of the best performing one from among these tests. For selection of lag length, we utilize MAIC proposed by $\mathrm{Ng}$ and Perron (2001). Simulation results for different serial correlation specifications will be provided by the authors upon request.
} 
Table 4

Empirical size and power with MA(2) innovations.

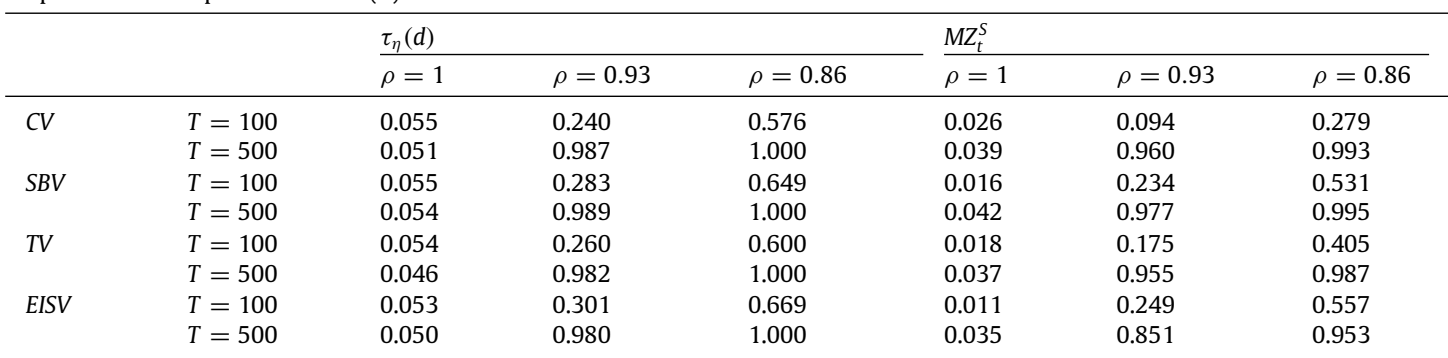

\section{Appendix}

Proof of Lemma 1. Part (i) can be found in Theorem 1 of Cavaliere and Taylor (2007) and Remark 3.1. Part (ii) is from Proposition 3 of Cavaliere (2005).

For part (iii), write the partial sum process for $\tilde{y}_{t}$ as follows:

$$
\begin{aligned}
\tilde{y}_{T}(t) & =T^{-d} \Delta_{+}^{-d} y_{T}(t)=T^{-1 / 2-d} \sum_{k=0}^{\lfloor T t\rfloor-1} \pi_{k}(d) y_{\lfloor T t\rfloor-k} \\
& =T^{-1 / 2-d} \sum_{k=1}^{\lfloor T t\rfloor} \pi_{\lfloor T t\rfloor-k}(d) y_{k}
\end{aligned}
$$

where $\pi_{k}(d)=\frac{\Gamma(k+d)}{\Gamma(d) \Gamma(k+1)}$, and from Wang et al. (2002) and Sowell (1990) we know that $\sum_{j=0}^{m} \pi_{j}(d)=\pi_{m}(d+1)$, thus we have:

$$
\begin{aligned}
& \tilde{y}_{T}(t)=T^{-1 / 2-d} \sum_{k=1}^{\lfloor T t\rfloor} \pi_{\lfloor T t\rfloor-k}(d) y_{k} \\
& =T^{-1 / 2-d} \sum_{k=1}^{\lfloor T t\rfloor} \pi_{\lfloor T t\rfloor-k}(d) \sum_{j=1}^{k} v_{j} \\
& =T^{-1 / 2-d} \sum_{k=1}^{\lfloor T t\rfloor} \sum_{j=1}^{k} \pi_{\lfloor T t\rfloor-k}(d) v_{j} \\
& =T^{-1 / 2-d} \sum_{k=1}^{\lfloor T t\rfloor} \pi_{\lfloor T t\rfloor-k}(d+1) v_{k} \\
& =T^{-1 / 2-d} \sum_{k=1}^{\lfloor T t\rfloor} \frac{(\lfloor T t\rfloor-k)^{d}}{\Gamma(d+1)} v_{k} \\
& =T^{-1 / 2} \sum_{k=1}^{\lfloor T t\rfloor} \frac{\left(\frac{\lfloor T t\rfloor-k}{T}\right)^{d}}{\Gamma(d+1)} v_{k} \\
& =T^{-1 / 2} \sum_{k=1}^{\lfloor T t\rfloor} \frac{(t-k / T)^{d}}{\Gamma(d+1)} v_{k} \\
& =\sum_{k=1}^{\lfloor T t\rfloor} \frac{(t-k / T)^{d}}{\Gamma(d+1)} T^{-1 / 2} \Delta y_{k} .
\end{aligned}
$$

Here we can define $y_{k}=\sum_{j=1}^{k} v_{k}$ in second equality of the first line. Note that $v_{k}=u_{k}$ for all $k$ when $c=0$. In the second line we utilize the above formula for fractional binomial coefficients. The third line involves basic operations for fractionally integrated series, which can be found in Nielsen (2009). Finally $v_{k}=\Delta y_{k}$. Here $\Delta y_{k}$ can be written as $\int_{(k-1) / T}^{k / T} d y_{T}(s)$ in the limit (see Phillips,
1987), where $y_{T}(s)$ is partial sum process for $y_{t}$. Then,

$$
\begin{aligned}
\tilde{y}_{T}(t) & =\sum_{k=1}^{\lfloor T t\rfloor} \frac{(t-k / T)^{d}}{\Gamma(d+1)} \int_{(k-1) / T}^{k / T} d y_{T}(s) \\
& =\sum_{k=1}^{\lfloor T\rfloor} \int_{(k-1) / T}^{k / T} \frac{(t-k / T)^{d}}{\Gamma(d+1)} d y_{T}(s) \\
& \stackrel{w}{\longrightarrow} \int_{0}^{t} \frac{(t-s)^{d}}{\Gamma(d+1)} d y_{T}(s) .
\end{aligned}
$$

Note that last equality comes from the fact that $s \in[(k-1) / T$, $(k / T)]$ and as $T \longrightarrow \infty(k-1) / T$ and $k / T$ will converge to the same limit, say $s$ in this case. Then, since $y_{T}(s)$ is continuous and converging to $J_{\omega}^{c}(s)$ according to Cavaliere and Taylor (2007) and $(t-s)^{d}$ is continuous, we can apply Continuous Mapping Theorem (CMT) to conclude that $\tilde{y}_{T}(t) \stackrel{w}{\longrightarrow} \frac{C(1)}{\Gamma(d+1)} \int_{0}^{t}(t-s)^{d} d J_{\omega}^{c}(s)$.

Proof of Theorem 1. To prove part (i), first consider the residuals from the regression of $\delta_{t}$ on $x_{t}$ for $t=1 \ldots T$, for $s \in[0,1]$ :

$$
\begin{aligned}
& \hat{x}_{\lfloor T s\rfloor}=y_{\lfloor T s\rfloor}-(\hat{\theta}-\theta)^{\prime} \delta_{\lfloor T S\rfloor} \\
& T^{-1 / 2} \hat{x}_{\lfloor T s\rfloor}=T^{-1 / 2} y_{\lfloor T S\rfloor}-T^{-1 / 2}(\hat{\theta}-\theta)^{\prime} \delta_{\lfloor T S\rfloor} .
\end{aligned}
$$

We have already establish limiting distribution for first factor on the right hand side of Eq. (16). For second factor, define $N_{1}(T)=1$ when $\delta_{t}=1$ and $N_{2}(T)=\left[\begin{array}{cc}1 & 0 \\ 0 & T^{-1}\end{array}\right]$ when $\delta_{t}=[1, t]^{\prime}$, we have same structure as in Nielsen (2009):

$$
\begin{aligned}
T^{-1 / 2}(\hat{\theta}-\theta)^{\prime} \delta_{\lfloor T S\rfloor} & \left(T^{-1} \sum_{r=1}^{T} T^{-1 / 2} y_{r} \delta_{r^{\prime}} N_{j}(T)\right) \\
& \times\left(T^{-1} \sum_{r=1}^{T} N_{j}(T) \delta_{r} \delta_{r^{\prime}} N_{j}(T)\right)^{-1} N_{j}(T) \delta_{\lfloor T s\rfloor} \\
= & \left(T^{-1} \sum_{r=1}^{T} T^{-1 / 2} y_{r} D_{j}(r / T)^{\prime}\right) \\
& \times\left(T^{-1} \sum_{t=1}^{T} D_{j}(r / T) D_{j}(r / T)^{\prime}\right)^{-1} D_{j}(\lfloor T s\rfloor / T)
\end{aligned}
$$

where $D_{j}(\lfloor T s\rfloor / T)=N_{j}(T) \delta_{s}$. Note that $D_{j}(\lfloor T s\rfloor / T) \longrightarrow D_{j}(s)$ and $D_{j}(s)$ is defined in Theorem 1 part (i). By application of lemmas (i-ii) and CMT, we have:

$$
\begin{aligned}
T^{-1 / 2}(\hat{\theta}-\theta) \delta_{\lfloor T s\rfloor} \stackrel{w}{\longrightarrow} & \bar{\omega} C(1)\left(\int_{0}^{1} J_{\eta}^{c}(r) D_{j}(r)^{\prime} d r\right) \\
& \times\left(\int_{0}^{1} D_{j}(r) D_{j}(r)^{\prime} d r\right)^{-1} D_{j}(s) .
\end{aligned}
$$


Finally we have

$$
\begin{aligned}
T^{-1 / 2} \hat{x}_{\lfloor T s\rfloor} \stackrel{w}{\longrightarrow} & \bar{\omega} C(1) J_{\eta}^{c}(s)-\bar{\omega} C(1)\left(\int_{0}^{1} J_{\eta}^{c}(r) D_{j}(r)^{\prime} d r\right) \\
& \times\left(\int_{0}^{1} D_{j}(r) D_{j}(r)^{\prime} d r\right)^{-1} D_{j}(s):=J_{\eta, j}^{c}(s) .
\end{aligned}
$$

For part (ii) first consider

$$
\begin{aligned}
\tilde{\hat{x}}_{t} & =\Delta_{+}^{-d} y_{t}-T^{-d} \Delta_{+}^{-d}(\hat{\theta}-\theta) \delta_{k} \\
& =\sum_{k=0}^{T} \pi_{k}(d) y_{t-k}-(\hat{\theta}-\theta) \sum_{k=0}^{T} \pi_{k}(d) \delta_{t-k} .
\end{aligned}
$$

We can write the partial sum process to find the limits

$$
\begin{aligned}
T^{-1 / 2-d} \tilde{\hat{x}}_{t}= & T^{-1 / 2-d} \sum_{k=0}^{T} \pi_{k}(d) y_{t-k} \\
& -T^{-1 / 2-d}(\hat{\theta}-\theta) \sum_{k=0}^{T} \pi_{k}(d) \delta_{t-k} .
\end{aligned}
$$

First factor converges by Lemma 1 part (iii). For the second factor write:

$T^{-1 / 2}(\hat{\theta}-\theta) T^{-d} \sum_{k=0}^{T} \pi_{k}(d) \delta_{t-k}$

here $T^{-1 / 2}(\hat{\theta}-\theta)^{\prime} \longrightarrow \bar{\omega} C(1)\left(\int_{0}^{1} J_{\eta}^{c}(r) D_{j}(r)^{\prime} d r\right)\left(\int_{0}^{1} D_{j}(r)\right.$

$\left.D_{j}(r)^{\prime} d r\right)^{-1}$ by Eq. (17).

The convergence for $T^{-d} \sum_{k=0}^{T} \pi_{k}(d) \delta_{t-k}$ is already proved by Nielsen (2009), that is

$T^{-d} \sum_{k=0}^{\lfloor T t\rfloor} \pi_{k}(d) \delta_{\lfloor T t\rfloor-k} \longrightarrow \int_{0}^{t} \frac{(t-s)^{d-1}}{\Gamma(d)} D_{j}(s) d s$

this establishes the proof.

Last part, (iii), is derived by application of CMT using the limits we found in parts (i)-(ii).

Proof of Theorem 2. Part (i) directly follows from Theorem 3 of Cavaliere and Taylor (2007).

For part (ii), define the process $s_{t}=\sum_{k=1}^{t} e_{k}$, the partial sum $S_{T}(t)=T^{-1 / 2} \sum_{k=1}^{\lfloor T t\rfloor} e_{k}$ and $\tilde{S}_{T}(t)=T^{-d} \Delta_{+}^{-d} S_{T}(t)$ where $e_{t}$ is i.i.d. $\mathrm{N}(0,1)$ for all $t$. Note that we can write:

$$
\begin{aligned}
\tilde{S}_{T}(t) & =T^{-d} \Delta_{+}^{-d} S_{T}(t)=T^{-1 / 2-d} \sum_{k=0}^{\lfloor T t\rfloor-1} \pi_{k}(d) s_{\lfloor T t\rfloor-k} \\
& =T^{-1 / 2-d} \sum_{k=1}^{\lfloor T t\rfloor} \pi_{\lfloor T t\rfloor-k}(d) s_{k} .
\end{aligned}
$$

This is not different than the partial sum process of $\tilde{y}_{t}$ in the proof of Lemma 1(iii), but we replace $y_{k}$ with $s_{k}$. Consequently, we can use same arguments here and obtain a similar expression as in Eq. (14):

$\tilde{S}_{T}(t) \stackrel{w}{\longrightarrow} \int_{0}^{t} \frac{(t-s)^{d}}{\Gamma(d+1)} d S_{T}(s)$.

Now, $B_{\hat{\eta}, T}(s):=S_{T}(\hat{\eta}((\lfloor T s\rfloor / T) T))$ as in CT and

$B_{\hat{\eta}, d, T}(s):=\tilde{S}_{T}(\hat{\eta}((\lfloor T s\rfloor / T) T))=T^{-d} \Delta_{+}^{-d} S_{T}(\hat{\eta}((\lfloor T s\rfloor / T) T))$

then replacing $s$ with $(\hat{\eta}((\lfloor T S\rfloor / T) T))$ in (18), we have:

$B_{\hat{\eta}, d, T}(s) \stackrel{w}{\longrightarrow} \int_{0}^{t} \frac{(t-s)^{d}}{\Gamma(d+1)} d S_{T}(\hat{\eta}((\lfloor T s\rfloor / T) T))$.

But, from part (i), Theorem 3 of Cavaliere and Taylor (2007) indicates that $\left(S_{T}(s), \hat{\eta}(s)\right)$ jointly converges to $(B(s), \eta(s))$, thus $S_{T}(\hat{\eta}((\lfloor T s\rfloor / T) T)) \stackrel{w}{\longrightarrow} B_{\eta}(s)$. Applying CMT with $d S_{T}(\hat{\eta}$ $((\lfloor T s\rfloor / T) T)) \stackrel{w}{\longrightarrow} d B_{\eta}(s)$, we obtain the result.

\section{References}

Busetti, Fabio, Taylor, AM. Robert, 2003. Testing against stochastic trend and seasonality in the presence of unattended breaks and unit roots. J. Econometrics 117 (1), 21-53.

Cavaliere, Giuseppe, 2005. Unit root tests under time-varying variances. Econometric Rev. 23 (3), 259-292.

Cavaliere, Giuseppe, Taylor, AM. Robert, 2007. Testing for unit roots in time series models with non-stationary volatility. J. Econometrics 140 (2), 919-947.

Demetrescu, Matei, Sibbertsen, Philipp, 2014. Inference on the long-memory properties of time series with non-stationary volatility. Technical report, Discussion Paper. Wirtschaftswissenschaftliche Fakultät, Leibniz University of Hannover.

Marinucci, Domenico, Robinson, Peter M., 2000. Weak convergence of multivariate fractional processes. Stochastic Process. Appl. 86 (1), 103-120.

McConnell, Margaret Mary, Perez-Quiros, Gabriel, 1998. Output fluctuations in the United States: what has changed since the early 1980s? FRB of New York Staff Report, 41.

$\mathrm{Ng}$, Serena, Perron, Pierre, 2001. Lag length selection and the construction of unit root tests with good size and power. Econometrica 1519-1554.

Nielsen, Morten Ørregaard, 2009. A powerful test of the autoregressive unit root hypothesis based on a tuning parameter free statistic. Econometric Theory 25 (06), 1515-1544.

Phillips, Peter C.B., 1987. Towards a unified asymptotic theory for autoregression. Biometrika 74 (3), 535-547.

Phillips, P.C.B., Perron, P., 1988. Testing for a unit root in time series regression. Biometrika 75 (2), 335-346.

Sensier, Marianne, Van Dijk, Dick, 2004. Testing for volatility changes in us macroeconomic time series. Rev. Econ. Stat. 86 (3), 833-839.

Sowell, Fallaw, 1990. The fractional unit root distribution. Econometrica 495-505.

Wang, Q., Lin, Y.X., Gulati, C., 2002. Asymptotics for general nonstationary fractionally integrated processes without prehistoric influence. J. Appl. Math. Decis. Sci. 6 (4), 255-269. 